\title{
Interior Symmetry and Local Bifurcation in Coupled Cell Networks
}

\author{
Martin Golubitsky \\ Department of Mathematics \\ University of Houston \\ Houston TX 77204-3476, USA
}

\author{
Marcus Pivato \\ Department of Mathematics \\ Trent University \\ Peterborough, ON, Canada K9L 1 Z6
}

Ian Stewart

Mathematics Institute

University of Warwick

Coventry CV4 7AL, UK

September 30, 2003

\begin{abstract}
A coupled cell system is a network of dynamical systems, or 'cells', coupled together. Such systems can be represented schematically by a directed graph whose nodes correspond to cells and whose edges represent couplings. A symmetry of a coupled cell system is a permutation of the cells that preserves all internal dynamics and all couplings. It is well known that symmetry can lead to patterns of synchronized cells, rotating waves, multirhythms, and synchronized chaos. Recently, the introduction of a less stringent form of symmetry, the 'symmetry groupoid', has shown that symmetry is not the only mechanism that can create such states in a coupled cell system. The symmetry groupoid consists of structure-preserving bijections between certain subsets of the cell network, the input sets.

Here we introduce a concept intermediate between the groupoid symmetries and the global group symmetries of a network: 'interior symmetry'. This concept is closely related to the groupoid structure, but imposes stronger constraints.

We develop the local bifurcation theory of coupled cell systems possessing interior symmetries, by analogy with symmetric bifurcation theory. The main results are analogs for 'synchrony-breaking' bifurcations of the Equivariant Branching Lemma for steady-state bifurcation, and the Equivariant Hopf Theorem for bifurcation to timeperiodic states.
\end{abstract}




\section{Introduction}

A coupled cell system is a a system of ordinary differential equations (ODE) whose structure is that of a finite number of subsystems ('cells') that are coupled together. That is, the dynamics of a given cell depends on the states of some of the other cells, as well as its own state. Which cells are coupled can be specified by a network or directed graph $G$, whose nodes are the cells and whose (directed) edges correspond to couplings. That is, cell $i$ influences cell $j$ if there is a directed edge from node $i$ to node $j$.

Abstractly, the topology of the network (especially when equipped with 'labels' specifying when two cells or two couplings are 'the same' [34]) determines a class $\mathcal{F}_{G}^{P}$ of 'admissible' vector fields. The corresponding ODEs are precisely the coupled cell systems whose 'architecture' is that of $G$. It turns out that many aspects of pattern-formation in coupled cell systems are primarily consequences of the network topology, and that the precise choice of an admissible vector field is of secondary significance.

At this level of generality, any dynamical system can be thought of as a coupled cell system, and conversely. However, there is a significant difference in viewpoint. A coupled cell system is equipped with a distinguished set of 'observables', the states of the cells. Specifically, let $\mathcal{C}=\{1, \ldots, N\}$ and let the cells be indexed by $c \in \mathcal{C}$. Equip each cell $c$ with a phase space $P_{c}$ (a finite-dimensional smooth manifold, which for simplicity we shall take to be $\mathbf{R}^{k}(c)$ where $k(c)$ is the number of 'degrees of freedom' of cell $c$ ). Then the total phase space for the system is

$$
P=P_{1} \times \cdots \times P_{N}
$$

and the state of the system at time $t$ is $\left(x_{1}(t), \ldots x_{N}(t)\right)$, where $x_{c}(t) \in P_{c}$ is the state of cell $c$ at time $t$. The distinguished observables are then the natural projections $\pi_{c}: P \rightarrow P_{c}$ from the total phase space to the phase spaces of the cells are an important part of the structure, so that, for example, the only permitted changes of variables are ones that preserve these projections, [23, 24].

The existence of distinguished observables makes it meaningful to compare the dynamics of distinct cells. For example we may say that cells $i, j$ are synchronous if $x_{i}(t)=x_{j}(t)$ for all $t \in \mathbf{R}$, or that they are phase-related with phase difference $\theta$ if both $x_{i}, x_{j}$ are time-periodic and $x_{j}(t)=x_{i}(t+\theta)$. There now exists a huge literature on synchrony, phase-relations, and more exotic dynamical phenomena in coupled cell systems, often referred to simply as 'networks': see for example Kuramoto [29], Pecora and Carroll [30], Boccaletti et al. [5], Wang [36], Watts and Strogatz [37]. The potential range of applications of such systems is also huge, including communication via the Internet, the spread of epidemics, food webs in ecosystems, metabolic networks in the cell, neural circuits, networks of gene expression, commercial supply chains, electrical power grids, transport networks, and crowd flow.

One strand in the literature focuses on the effect of the overall symmetry of the network on the formation of spatial and spatiotemporal patterns [16, 17, 23, 24]. Applications include animal locomotion $[7,8,9,10,11,12,26]$ and speciation $[13,18,19,20,14,31,32,33]$. A continuum analog has been applied to visual perception $[2,3,4]$.

The formal setting for this theory is the symmetry group of the network: the set of 
permutations of the nodes that preserves the coupling structure. However, the existence of global network symmetries is a very strong constraint, and it turns out that for many purposes a weaker notion is preferable, the symmetry groupoid of the network [34, 21, 25].

A groupoid is a generalization of a group, in which products of elements are not always defined (Higgins [28]). The symmetry groupoid is a formalization of the 'local symmetries' of a coupled cell network that relate subsets of the network to each other. In particular, the 'admissible' vector fields — those specified by the network topology — are precisely those that are equivariant under the action of the symmetry groupoid [34].

In this paper we study a concept of 'symmetry' that is intermediate between the global group symmetries of $G$ and the local groupoid symmetries. We refer to this concept as interior symmetry. Roughly speaking, an interior symmetry is a symmetry of some subset of cells, that fixes all cells that receive inputs from cells outside that subset. Interior symmetries have a strong influence on pattern formation, and the details of this influence differ from both the group and groupoid cases.

Our main objective in this paper is to initiate the 'synchrony-breaking' bifurcation theory of not necessarily symmetric coupled cell systems in the presence of interior symmetries, and in particular to prove analogs of the Equivariant Branching Lemma and the Equivariant Hopf Theorem [27, 23]. Section 2 reviews the pertinent features of coupled cell systems and their associated networks. In particular we give a formal definition of a coupled cell network, explain its graphical representation, discuss admissible vector fields, and recall the concept of a balanced equivalence relation. Section 3 defines interior symmetries and establishes some of their properties. Section 4 sets up the required properties of linearizations. Section 5 states and proves an analog of the Equivariant Branching Lemma, while Section 6 states and proves an analog of the Equivariant Equivariant Hopf Theorem. We conclude with a typical simulation for the time-periodic case.

\section{Coupled Cell Systems and Network Topology}

We recall the coupled cell network formalism. There are two versions: the original version of [34], and the 'multiple-arrow' generalization of [25], which has important technical advantages (at the expense of slight complications in the definition of a network). We use the multiplearrow version.

\section{Formal Definition of a Coupled Cell Network}

Definition 2.1 A coupled cell network $\mathcal{G}$ consists of:

(a) A finite $\operatorname{set} \mathcal{C}=\{1, \ldots, N\}$ of nodes or cells.

(b) An equivalence relation $\sim_{C}$ on cells in $\mathcal{C}$.

The type or cell label of cell $c$ is the $\sim_{C^{-}}$equivalence class $[c]_{C}$ of $c$. 
(c) Associated to each node $c$ is a finite set of input terminals $I(c)$. Each input terminal $i \in I(c)$ is the receptacle for one arrow or edge that begins in tail cell $\tau(i)$ and ends in terminal $i$. That arrow is denoted by $(\tau(i), i)$, has head cell $c$, and head terminal $i$. Let $\mathcal{E}$ denote the set of all arrows.

(d) An equivalence relation $\sim_{E}$ on edges in $\mathcal{E}$.

The type or coupling label of edge $e$ is the $\sim_{E}$-equivalence class $[e]_{E}$ of $e$.

(e) Equivalent edges have equivalent tails and heads. That is, if $(\tau(i), i) \sim_{E}(\tau(j), j)$ where $i \in I(c)$ and $j \in I(d)$, then $\tau(i) \sim_{C} \tau(j)$ and $c \sim_{C} d$.

Definition 2.2 The relation $\sim_{I}$ of input equivalence on $\mathcal{C}$ is defined by $c \sim_{I} d$ if and only if there exists a bijection

$$
\beta: I(c) \rightarrow I(d)
$$

that preserves arrows, in the sense that for every $i \in I(c)$,

$$
(\tau(i), i) \sim_{E}(\tau(\beta(i)), \beta(i))
$$

Such a bijection $\beta$ is an input isomorphism from $c$ to $d$. Let $B(c, d)$ denote the set of all input isomorphisms from $c$ to $d$. Then the (disjoint) union

$$
\mathcal{B}_{\mathcal{G}}=\bigcup_{c, d \in \mathcal{C}} B(c, d)
$$

is a groupoid (Brandt [1], Brown [6], Higgins [28]). We call $\mathcal{B}_{\mathcal{G}}$ the groupoid of the network. $\diamond$

\section{Graphical Representation}

We can represent abstract classes of networks with the same general 'architecture' by labeled directed graphs. Figure 1 shows a simple example.

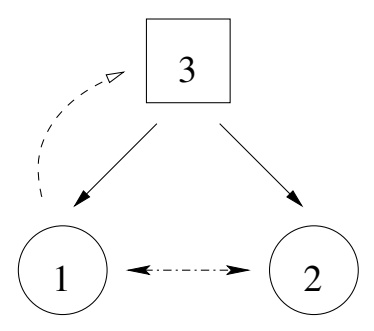

Figure 1: A three-cell network without symmetry.

Here the two circles and the square indicate cells; the symbols show that cells 1 and 2 have the same cell type, whereas cell 3 is different. There are three types of edges, the arrows. 
These occur in three edge-types: two identical arrows from cell 3 to cells 1,2 respectively; two identical arrows between cells 1 and 2, one in each direction; an arrow from cell 1 to cell 3 .

We now explain how to interpret such a diagram as being represenative of a class of vector fields.

\section{Admissible Vector Fields}

We now define a class $\mathcal{F}_{G}^{P}$ of 'admissible' vector fields corresponding to a given coupled cell network $\mathcal{G}$ on a total phase space $P$. For each cell in $\mathcal{C}$ select a cell phase space $P_{c}$. This is a smooth manifold of dimension $\geq 1$, assumed for simplicity to be a nonzero finite-dimensional vector space over $\mathbf{R}$. We require

$$
c \sim_{C} d \quad \Longrightarrow \quad P_{c}=P_{d}
$$

and we employ the same coordinate systems on $P_{c}$ and $P_{d}$. The corresponding total phase space is

$$
P=\prod_{c \in \mathcal{C}} P_{c}
$$

with coordinate system

$$
x=\left(x_{c}\right)_{c \in \mathcal{C}}
$$

More generally, let $\mathcal{D}=\left\{d_{1}, \ldots, d_{s}\right\}$ be any finite $s$-tuple of cells in $\mathcal{C}$ (so that in particular the same cell can appear more than once in $\mathcal{D}$ ). Define

$$
P_{\mathcal{D}}=P_{d_{1}} \times \cdots \times P_{d_{s}}
$$

Further, write

$$
x_{\mathcal{D}}=\left(x_{d_{1}}, \ldots, x_{d_{s}}\right)
$$

where $x_{d_{j}} \in P_{d_{j}}$.

Suppose that $\mathcal{D}_{1}, \mathcal{D}_{2}$ are subsets of $\mathcal{C}$, and that there is a bijection $\gamma: \mathcal{D}_{1} \rightarrow \mathcal{D}_{2}$ such that $\gamma(d) \sim_{C} d$ for all $d \in \mathcal{D}_{1}$. Define the pullback map

$$
\gamma^{*}: P_{\mathcal{D}_{2}} \rightarrow P_{\mathcal{D}_{1}}
$$

by

$$
\left(\gamma^{*}(z)\right)_{j}=z_{\gamma(j)}
$$

for all $j \in \mathcal{D}_{1}$ and $z \in P_{\mathcal{D}_{2}}$. Let the coupling phase space of cell $c$ be

$$
P_{\tau(I(c))}=P_{\tau\left(i_{1}\right)} \times \cdots \times P_{\tau\left(i_{s}\right)}
$$

Definition 2.3 A vector field $f: P \rightarrow P$ is $\mathcal{B}_{G^{-}}$equivariant or $G$-admissible if: 
(a) For all $c \in \mathcal{C}$ the component $f_{c}(x)$ depends only on the internal phase space variables $x_{c}$ and the coupling phase space variables $x_{\tau(I(c))}$; that is, there exists $\hat{f}_{c}: P_{c} \times P_{\tau(I(c))} \rightarrow P_{c}$ such that

$$
f_{c}(x)=\hat{f}_{c}\left(x_{c}, x_{\tau(I(c))}\right)
$$

(b) For all $c, d \in \mathcal{C}$ and $\beta \in B(c, d)$

$$
\hat{f}_{d}\left(x_{d}, x_{\tau(I(d))}\right)=\hat{f}_{c}\left(x_{d}, \beta^{*}\left(x_{\tau(I(d))}\right)\right)
$$

for all $x \in P$.

Observe that self-coupling is allowed (that is, $P_{c}$ can be one of the factors in $P_{\tau(I(c))}$ ) and multiple arrows between two cells are allowed (since the tail of two arrows terminating in $I(c)$ can be the same cell). However, when repetition occurs, the repeated coordinates are identical.

As in [34] Lemma 4.5, any $\mathcal{B}_{G^{-}}$equivariant vector field $F$ is determined by one mapping $f$ for each input equivalence class of cells. Each component $f_{c}$ of $F$ is invariant under the vertex group $B(c, c)$. Indeed, if we choose once such invariant function for one $c$ in each

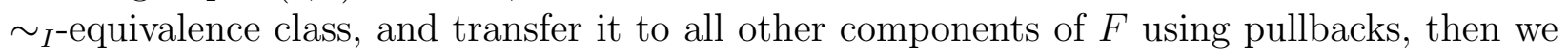
have specified a unique admissible vector field.

Example 2.4 Consider the network of Figure 1. Cell phase spaces $P_{1}$ and $P_{2}$ are identical and equal to $\mathbf{R}^{k}$, whereas $P_{3}=\mathbf{R}^{\ell}$ is not necessarily the same. Coordinates on $P$ take the form $\left(x_{1}, x_{2}, x_{3}\right)$. Admissible vector fields are those of the form

$$
\begin{aligned}
\dot{x_{1}} & =f\left(x_{1}, x_{2}, x_{3}\right) \\
\dot{x_{2}} & =f\left(x_{2}, x_{1}, x_{3}\right) \\
\dot{x_{3}} & =g\left(x_{3}, x_{1}\right)
\end{aligned}
$$

where $x_{1}, x_{2} \in \mathbf{R}^{k}$ and $x_{3} \in \mathbf{R}^{\ell}$.

If the arrows between cells 1 and 2 are deleted, this network is the 'master/slave' system of Pecora and Carroll [30] equation (3) with $\left(x_{1}, x_{2}, x_{3}\right)=\left(w, w^{\prime}, v\right)$. They study synchronization of $w, w^{\prime}$; that is, of cells 1 and 2 . We now look at why such synchronization is reasonable.

\section{Balanced Equivalence Relations}

Finally, we formalize the concept of synchrony. We adopt a strong definition: cells $c, d$ are synchronous if $x_{c}(t)=x_{d}(t)$ for all $t \in \mathbf{R}$. This condition depends on the dynamic trajectory $x(t)$; that is, it depends on initial conditions. The point of view is that the first step in understanding patterns of synchrony is to consider existence issues. Questions such as the dynamic stability of the synchronous state, or various weaker forms of synchrony, can then be placed in context. 
Abstractly, the relation ' $c$ and $d$ are synchronous' is an equivalence relation on the set of cells, so we formalize patterns of synchrony in terms of an equivalence relation $\bowtie$ on $\mathcal{C}$. We assume that $\bowtie$ is a refinement of $\sim_{\mathcal{C}}$; that is, if $c \bowtie d$, then $c$ and $d$ have the same cell labels. (Otherwise 'synchronous' makes no sense.) The corresponding polydiagonal subspace (or just polydiagonal) is

$$
\Delta_{\bowtie}=\left\{x \in P: x_{c}=x_{d} \text { whenever } c \bowtie d \quad \forall c, d \in \mathcal{C}\right\}
$$

This space is well-defined since $x_{c}$ and $x_{d}$ lie in the same space $P_{c}=P_{d}$. The polydiagonal $\Delta_{\bowtie}$ is a linear subspace of $P$. It is precisely the subspace on which all cells in the same $\bowtie$-equivalence class are synchronous.

Patterns of robust synchrony are classified by a special type of equivalence relation:

Definition 2.5 An equivalence relation $\bowtie$ on $\mathcal{C}$ is balanced if for every $c, d \in \mathcal{C}$ with $c \bowtie d$, there exists an input isomorphism $\beta \in B(c, d)$ such that $\tau(i) \bowtie \tau(\beta(i))$ for all $i \in I(c)$.

In particular, $B(c, d) \neq \emptyset$ implies $c \sim_{I} d$. Hence, balanced equivalence relations refine input equivalence $\sim_{I}$.

Definition 2.6 Let $\bowtie$ be an equivalence relation on $\mathcal{C}$ that refines $\sim_{C}$. Then $\bowtie$ is robustly polysynchronous if $\Delta_{\bowtie}$ is invariant under every vector field $f \in \mathcal{F}_{G}^{P}$. That is,

$$
f\left(\Delta_{\bowtie}\right) \subseteq \Delta_{\bowtie}
$$

for all $f \in \mathcal{F}_{G}^{P}$. Equivalently, if $x(t)$ is a trajectory of any $f \in \mathcal{F}_{G}^{P}$, with initial condition $x(0) \in \Delta_{\bowtie}$, then $x(t) \in \Delta_{\bowtie}$ for all $t \in \mathbf{R}$.

The main theorem on balanced equivalence relations is Theorem 4.3 of [25]:

Theorem 2.7 Let $\bowtie$ be an equivalence relation on a coupled cell network. Then $\bowtie$ is robustly polysynchronous if and only if $\bowtie$ is balanced.

Example 2.8 We continue with our running example, the 3-cell network of Figure 1. There is an equivalence relation $\bowtie$ for which $1 \bowtie 2$; its equivalence classes are $\{1,2\}$ and $\{3\}$. The corresponding polydiagonal is $\Delta_{\bowtie}=\left\{x: x_{1}=x_{2}\right\}=\{(x, x, y)\}$. On this subspace the differential equations become

$$
\begin{aligned}
\dot{x} & =f(x, x, y) \\
\dot{x} & =f(x, x, y) \\
\dot{y} & =g(y, x)
\end{aligned}
$$

Since the first two equations are identical, $\Delta_{\bowtie}$ is invariant under all admissible vector fields. Thus the synchrony is 'robust'.

The relation $\bowtie$ is also balanced. The only condition to verify is that cells 1 and 2 , which are $\bowtie$-equivalent but distinct, have input sets that are isomorphic by an isomorphism that preserves $\bowtie$-equivalence classes. The input sets are

$$
I(1)=\{1,2,3\} \quad I(2)=\{2,1,3\}
$$

where as a convention we list the 'base point' $c$ of $I(c)$ first. The permutation $\beta: I(1) \rightarrow I(2)$ with $\beta(1)=2, \beta(2)=1, \beta(3)=3$ is an input isomorphism that preserves $\bowtie$-equivalence classes. That is, $\bowtie$ is balanced as claimed. 


\section{Interior Symmetries and Local Bifurcation Theory}

With these preliminaries out of the way, we now turn to the main aim of this paper, which is to analyze some special types of local bifurcation in coupled cell systems: synchronybreaking bifurcations. These occur when a synchronous state loses stability and bifurcates to a state with less synchrony. Such bifurcations can be considered to be a generalization of symmetry-breaking bifurcations in symmetric coupled cell systems.

In the context of symmetry-breaking, there are two main local bifurcation theorems [27, 23]. The Equivariant Branching Lemma ([27] Chapter XIII Section 3) proves the existence of certain branches of symmetry-breaking steady states; the Equivariant Hopf Theorem ([27] Chapter XVI Section 4) proves the existence of certain branches of spatio-temporal symmetry-breaking time-periodic states. We will prove analogs of both of these theorems for coupled cell systems in a groupoid-equivariant setting. The analog of the Equivariant Branching Lemma is a natural generalization of the symmetric case, but the analogue of the Equivariant Hopf Theorem has novel and rather restrictive features. In particular, instead of proving the existence of states with certain spatio-temporal symmetries, we prove the existence of states whose linearizations on certain subsets of cells, near bifurcation, are superpositions of synchronous states with states having spatio-temporal symmetries.

The main concept involved in both bifurcation theorems is the notion of an 'interior symmetry'. Roughly, this is a permutation of cells that preserves a certain amount of input structure. Interior symmetries are related to the symmetry groupoid, but in general do not belong to it.

Recall $[27,23]$ that in general a symmetry $\gamma$ of a differential equation

$$
\dot{x}=f(x)
$$

with phase space $X=\mathbf{R}^{k}$ is a linear map $x \mapsto \gamma x$ on $X$ that commutes with $f$; that is, $\gamma$ satisfies

$$
f(\gamma x)=\gamma f(x) \quad \forall x \in X
$$

Let $x_{0}$ be an equilibrium that is fixed by $\gamma$. Then the Jacobian of $f$ at $x_{0}$ commutes with $\gamma$; that is,

$$
(d f)_{x_{0}} \gamma=\gamma(d f)_{x_{0}}
$$

This commutativity can force $(d f)_{x_{0}}$ to have multiple eigenvalues [27] and is the source of many of the complications in equivariant bifurcation theory. However, it can also help resolve those complications. Similar problems arise in the context of interior symmetries, and again the structure that causes them can sometimes be used to resolve them.

Throughout this section we work with a coupled cell network $G$ on a set $\mathcal{C}$ of $N$ nodes, and use the same notation as before. In particular, $f$ is a $G$-admissible vector field on a total phase space $P$. The symmetries that concern us here are permutations of the cells.

First, we relate the symmetry group of a coupled cell network to its symmetry groupoid. Suppose that a permutation $\sigma$ is a symmetry of the coupled cell system. Then it is easy to show that for each cell $j$,

$$
\left.\sigma\right|_{I(j)}: I(j) \rightarrow I(\sigma(j)) \text { is an input isomorphism. }
$$


Moreover, it is easy to see that the converse is also true. That is, if $\sigma$ satisfies (3.1) for every cell $j$, then $\sigma$ is a symmetry. The proof is a direct application of $\mathcal{B}_{G^{-}}$equivariance.

We now introduce an important concept for the local bifurcation theory of coupled cell networks:

Definition 3.1 Let $\mathcal{S} \subseteq \mathcal{C}$ be a subset of cells, and let $\sigma$ be a permutation of $\mathcal{C}$ that is the identity on the complement $\mathcal{C} \backslash \mathcal{S}$ of $\mathcal{S}$. Then $\sigma$ is an interior symmetry on $\mathcal{S}$ if $\sigma$ satisfies (3.1) for every $j \in \mathcal{S}$.

The interior symmetry group $\Sigma_{\mathcal{S}}$ is the set of all interior symmetries on $\mathcal{S}$. It is obviously a group.

The interior symmetry group $\Sigma_{\mathcal{C}}$ on the whole network $\mathcal{C}$ is the usual symmetry group $\Gamma$ of the entire coupled cell system.

Example 3.2 We now relate our running example, the three-cell network of Figure 1, to interior symmetries. Because of the (dotted) arrow from cell 1 to cell 3, the permutation (1 2) $\in \mathbf{S}_{3}$ is not a group symmetry of the network, but it is an interior symmetry on the subset $\mathcal{S}=\{1,2\}$.

A state $\left(x_{1}, x_{2}, x_{3}\right)$ is fixed by this interior symmetry if and only if $x_{1}=x_{2}$, that is, cells 1 and 2 are synchronous. That is, they lie in the polydiagonal $\Delta_{\bowtie}=\left\{x: x_{1}=x_{2}\right\}=$ $\{(x, x, y)\}$ corresponding to the balanced equivalence relation $\bowtie$ whose equivalence classes are $\{1,2\},\{3\}$.

In the context of interior symmetry we can characterize the polydiagonal group-theoretically, as a fixed-point subspace. Specifically, groupoid-equivariance of the cell system implies that

$$
f_{\mathcal{S}}\left(\sigma x_{\mathcal{S}}, x_{\mathcal{C} \backslash \mathcal{S}}\right)=\sigma f_{\mathcal{S}}\left(x_{\mathcal{S}}, x_{\mathcal{C} \backslash \mathcal{S}}\right)
$$

where $f_{\mathcal{S}}$ is the cell system vector field on the cells in $\mathcal{S}$ and $\sigma \in \Sigma_{\mathcal{S}}$.

Suppose that $\mathrm{T} \subset \Sigma_{\mathcal{S}}$ is a subgroup. Then

$$
\operatorname{Fix}(\mathrm{T})=\left\{\left(x_{\mathcal{S}}, x_{\mathcal{C} \backslash \mathcal{S}}\right) \in P: \delta x_{\mathcal{S}}=x_{\mathcal{S}} \quad \forall \delta \in \mathrm{T}\right\}
$$

Proposition 3.3 Let $\mathrm{T}$ be a subgroup of $\Sigma_{\mathcal{S}}$, and let $f$ be a $G$-admissible vector field. Then the subspace $\operatorname{Fix}(\mathrm{T})$ is flow-invariant for $f$.

Proof The equivalence relation on cells defined by $i \bowtie j$ if $i, j \in \mathcal{S}$ lie on the same $\mathrm{T}$ orbit is a balanced equivalence relation. Apply [34, Theorem 6.5] to show that the subspace $\Delta_{\bowtie}$ is flow-invariant where $\Delta_{\bowtie}$ is defined prior to [34, Definition 6.1]. Finally note that $\operatorname{Fix}(\mathrm{T})=\Delta_{\bowtie}$.

For related observations see Dias and Stewart [15] 


\section{Linear Theory}

Interior symmetries induce extra structure on linearized eigenvalues, and this structure controls the synchrony-breaking local bifurcations.

Example 4.1 The network of Figure 1 has the admissible vector fields in (2.6). Let $x_{0}=$ $\left(x_{1}, x_{1}, x_{3}\right)$ be a synchronous equlibrium and let $J$ be the Jacobian matrix at that equilibrium. Then

$$
J=\left[\begin{array}{lll}
a & b & c \\
b & a & c \\
d & 0 & e
\end{array}\right]
$$

where $a, b$ are $k \times k$ matrices, $c$ is a $k \times \ell$ matrix, $d$ is an $\ell \times k$, and $e$ is an $\ell \times \ell$ matrix.

In this example $\mathcal{S}=\{1,2\}, \Sigma_{\mathcal{S}}=\mathbf{Z}_{2}$, and $\operatorname{Fix}\left(\Sigma_{\mathcal{S}}\right)=\mathbf{R}\{(1,1,0),(0,0,1)\}$. Note that $W=\mathbf{R}\{(1,-1,0)\}$ is a complentary subspace to $\operatorname{Fix}\left(\Sigma_{\mathcal{S}}\right)$ on which $\Sigma_{\mathcal{S}}$ acts nontrivially (as $-I)$. Since $\operatorname{Fix}\left(\Sigma_{\mathcal{S}}\right)$ is flow-invariant, the matrix $\mathrm{J}$ written in the decomposition $W \oplus \operatorname{Fix}\left(\Sigma_{\mathcal{S}}\right)$ as $\hat{J}$ is block lower triangular. Indeed

$$
\hat{J}=\left[\begin{array}{c|cc}
a-b & 0 & 0 \\
\hline 0 & a+b & c \\
d & d & e
\end{array}\right]
$$

Thus synchrony-breaking bifurcations occur when the eigenvalues of $J \mid W$ are critical (that is, the $k \times k$ matrix $a-b$ has zero real part eigenvalues). In (4.4) and Lemma 4.2 (a) we show that this kind of decomposition occurs generally for linearizations about a synchronous equilibrium supported by an interior symmetry group.

We discuss steady-state and Hopf bifurcations from 'polysynchronous' equilibria, that is, equilibria in $\operatorname{Fix}\left(\Sigma_{\mathcal{S}}\right)$, that break interior symmetry.

The action of the group $\Sigma_{\mathcal{S}}$ decomposes $\mathcal{S}$ as

$$
\mathcal{S}=\mathcal{S}_{1} \cup \cdots \cup \mathcal{S}_{k}
$$

where each $\mathcal{S}_{j}$ is an orbit of the action. Let

$$
W=\left\{x \in P: x_{j}=0 \quad \forall j \in \mathcal{C} \backslash \mathcal{S} \quad \text { and } \quad \sum_{i \in \mathcal{S}_{\ell}} x_{i}=0 \quad \text { for } 1 \leq \ell \leq k\right\}
$$

Note that $W$ is a $\Sigma_{\mathcal{S}}$-invariant subspace.

We can write the state space $P$ as

$$
P=W \oplus \operatorname{Fix}\left(\Sigma_{\mathcal{S}}\right)
$$

In particular, (4.1) implies that vectors in $W$, when written in coupled cell coordinates, have zero components on all cells not in $\mathcal{S}$. 
Bifurcation theory concerns changes in solutions of an ODE as parameters are varied, so we introduce an explicit bifurcation parameter $\lambda \in \mathbf{R}$. We assume that $f$ (hence also its components $f_{c}$ ) depend on $\lambda$, and that the ODE

$$
\dot{x}=f(x, \lambda)
$$

has a 'trivial' equilibrium $x_{0} \in \operatorname{Fix}\left(\Sigma_{\mathcal{S}}\right)$. In the present context, we may assume without loss of generality that

$$
f\left(x_{0}, \lambda\right) \equiv 0
$$

and that the bifurcation occurs at $\lambda=0$. Let $L=(d f)_{x_{0}}$. Proposition 3.3 implies that $\operatorname{Fix}\left(\Sigma_{\mathcal{S}}\right)$ is invariant under $L$, so that $L$ has the block form

$$
L=\left[\begin{array}{ll}
A & 0 \\
C & B
\end{array}\right]
$$

with respect to the decomposition (4.2); that is, $A: W \rightarrow W$ and $B: \operatorname{Fix}\left(\Sigma_{\mathcal{S}}\right) \rightarrow \operatorname{Fix}\left(\Sigma_{\mathcal{S}}\right)$. Thus the eigenvalues of $L$ are the eigenvalues of $A$, together with those of $B$.

Local bifurcation (steady-state or Hopf) occurs when some eigenvalue of $L$ has zero real part. That eigenvalue is either associated with $A$ or with $B$, and it is the former case that concerns us here. We say that $f$ undergoes a bifurcation at $x_{0}$ that breaks interior symmetry if $A$ has an eigenvalue with zero real part. In this case, steady-state bifurcation occurs when $A$ has a zero eigenvalue, and Hopf bifurcation occurs when $A$ has a conjugate pair of purely imaginary eigenvalues. In the Hopf case, we may assume (after rescaling time if necessary) that the purely imaginary eigenvalues of $A$ are $\pm i$. We may also assume that the center subspace $E(A)$ is equal to $\operatorname{ker} A$ in steady-state bifurcation, and to the real eigenspace

$$
E(A)=\left\{x \in P:\left(A^{2}+1\right) x=0\right\}
$$

in Hopf bifurcation.

The structure of $L$ in (4.4) has several important implications:

Lemma 4.2 (a) A commutes with the action of $\Sigma_{\mathcal{S}}$ on $W$.

(b) A vector $u \in \operatorname{Fix}\left(\Sigma_{\mathcal{S}}\right)$ is an eigenvector of $B$ with eigenvalue $\mu$ if and only if $u$ is an eigenvector of $L$ with eigenvalue $\mu$.

(c) If $w \in W$ is an eigenvector of $A$ with eigenvalue $\mu$, then there exists an eigenvector $v$ of $L$ with eigenvalue $\mu$ of the form

$$
v=w+u
$$

where $u \in \operatorname{Fix}\left(\Sigma_{\mathcal{S}}\right)$.

(d) The center subspace $E(A)$ is $\Sigma_{\mathcal{S}}$-invariant. 
Proof Part (a) follows from Proposition 3.3 and the chain rule. Parts (b,c) are consequences of the block form of $L$. Part (d) follows because part (a) implies that all eigenspaces of $A$ are $\Sigma_{\mathcal{S}}$-invariant.

In our bifurcation studies we shall assume:

1) Critical eigenvalues $\mu$ ( 0 in steady-state bifurcation and $\pm i$ in Hopf bifurcation) extend uniquely and smoothly to eigenvalues $\mu(\lambda)$ for $\lambda$ near 0 .

2) The eigenvalue crossing condition

$$
\frac{d}{d \lambda} \operatorname{Re}(\mu)(0) \neq 0
$$

is valid.

\section{$5 \quad$ Interior Symmetry Branching Lemma}

Recall $[16,17,23]$ that an axial subgroup of a group action on a vector space is an isotropy subgroup whose fixed-point subspace is one-dimensional. We can now prove a generalization to coupled cell systems of the Equivariant Branching Lemma:

Theorem 5.1 Assume that $\operatorname{ker} L$ and $\operatorname{ker} A$ have the same dimension. Let $\mathrm{T} \subset \Sigma_{\mathcal{S}}$ be an axial subgroup of the action of $\Sigma_{\mathcal{S}}$ on $\operatorname{ker} A$, and assume the eigenvalue crossing condition (4.5). Then there exists a unique branch of equilibria, bifurcating from $\left(x_{0}, 0\right)$, with symmetry group $\mathrm{T}$.

Proof By Proposition 3.3, Fix(T) is flow-invariant. Since $\mathrm{T}$ is an axial subgroup, $\operatorname{ker} A \cap$ $\operatorname{Fix}(T)$ is one-dimensional and the bifurcation of $\left.f\right|_{\operatorname{Fix}(T) \times \mathbf{R}}$ is a simple eigenvalue bifurcation. Moreover, since $\left.f\right|_{\operatorname{Fix}\left(\Sigma_{\mathcal{S}}\right) \times \mathbf{R}}$ is nonsingular at $\lambda=0$, there is a unique branch of trivial solutions to $f=0$ in $\operatorname{Fix}\left(\Sigma_{\mathcal{S}}\right) \times \mathbf{R}$. By the usual implicit function theorem argument [27], the eigenvalue crossing condition implies the existence of a unique branch of nontrivial solutions to $f=0$ in $\operatorname{Fix}(T) \times \mathbf{R}$.

Remark 5.2 Liapunov-Schmidt reduction ([22] Chapter I Section 3) applied to the equation $f=0$ yields a reduced equation $g=0$, where

$$
g: \operatorname{ker} L \times \mathbf{R} \rightarrow \operatorname{ker} L
$$

whose solutions are in one-to-one correspondence with the solutions of $f=0$ near the origin. Even though Theorem 5.1 proves the simultaneous existence of branches of solutions to $g=0$ corresponding to each axial subgroup $\mathrm{T}$, the reduced mapping $g$ satisfies no obvious symmetry constraints. In particular:

1) The group $\Sigma_{\mathcal{S}}$ does not act (naturally) on ker $L$, even though it does act on ker $A$. 
2) The bifurcating branches can be transcritical, even when the symmetry condition $N_{\Sigma_{\mathcal{S}}}(\mathrm{T}) / \mathrm{T} \cong \mathbf{Z}_{2}$ (where $N_{\Sigma_{\mathcal{S}}}(\mathrm{T})$ is the normalizer of $\mathrm{T}$ in $\Sigma_{\mathcal{S}}$, see [27]) would imply that generic equivariant bifurcations are pitchfork bifurcations.

An example illustrating the second point is the 3-cell network of Figure 1, where we assume that each cell has one-dimensional internal dynamics. In this example, $\mathcal{S}=\{1,2\}$ and $\Sigma_{\mathcal{S}}=\mathbf{Z}_{2}(12)$. Generically, the bifurcating branch is transcritical. To ensure this, it is enough to assume that the coupling from cell 1 to cell 3 is nonzero at linear level.

\section{Interior Symmetry Hopf Theorem}

We now generalize the Equivariant Hopf Theorem ([27] Chapter XVI) to the context of interior symmetries of coupled cell systems. We begin this process by recalling the Equivariant Hopf Theorem.

Let $x(t)$ be a $2 \pi$-periodic solution of a system of ODEs with symmetry group $\Gamma$. A spatio-temporal symmetry of $x(t)$ is a pair $(\gamma, \theta) \in \Gamma \times \mathbf{S}^{1}$ such that

$$
\gamma x(t)=x(t+\theta)
$$

Here $\mathbf{S}^{1}$ is the circle group of phase shifts modulo the period. The group of (spatio-temporal) symmetries of a periodic solution is a subgroup $\Delta \subseteq \Gamma \times \mathbf{S}^{1}$. The subgroup $K=\Delta \cap \Gamma$ consists of the purely spatial symmetries of $x(t)$. It is known that $K$ must be an isotropy subgroup of the $\Gamma$-action $[27,9,23]$.

Consider now the context of $\Gamma$-equivariant vector fields $F: \mathbf{R}^{n} \times \mathbf{R} \rightarrow \mathbf{R}^{n}$ where $\Gamma$ is a (compact Lie) group, so that

$$
F(\gamma x, \lambda)=\gamma F(x, \lambda) \quad \forall \gamma \in \Gamma
$$

Assume that $F$ has a $\Gamma$-invariant equilibrium $x_{0}$ for all $\lambda$, that is,

$$
F\left(x_{0}, \lambda\right) \equiv 0
$$

Suppose that $x_{0}$ undergoes a Hopf bifurcation at $\lambda=0$. After rescaling time, we may suppose that

$$
L=\left(d_{x} F\right)_{x_{0}}
$$

has eigenvalues that include $\pm i$ when $\lambda=0$. Note that $L$ commutes with $\Gamma$ since $x_{0}$ is a $\Gamma$-invariant equilibrium.

There is a natural action of $\mathbf{S}^{1}$ on $E(L)$ given by the exponential $\exp (s L)$, and this action commutes with $\Gamma$ since $L$ commutes with $\Gamma$. Recall $[16,17,23]$ that an isotropy subgroup $\Delta \subset \Gamma \times \mathbf{S}^{1}$ is $\mathbf{C}$-axial if

$$
\operatorname{dim} \operatorname{Fix}_{E(L)}(\Delta)=2
$$

Suppose that the eigenvalue $\mu$ that extends $i$ at $\lambda=0$ crosses the imaginary axis with nonzero speed as $\lambda$ varies. Then the Equivariant Hopf Theorem states that for each $\mathbf{C}$-axial 
subgroup $\Delta$, there is a branch of periodic solutions emanating from $\left(x_{0}, 0\right)$ with spatiotemporal symmetry group $\Delta$. The proof uses Liapunov-Schmidt reduction in the context of loop spaces: see [27] Chapter XIV Section 4.

We now prove a generalization: for each $\mathbf{C}$-axial subgroup $\Delta$ of the action of a group of interior symmetries on $\mathcal{C}$, there is also a branch of periodic solutions emanating from this bifurcation point, whose structure is related to $\Delta$, but in a less straightforward way.

\section{Hopf Theorem with Interior Symmetry}

We pursue the same proof strategy to derive an analog of the Equivariant Hopf Theorem for interior symmetries. Let $G$ be a coupled cell network, let $\mathcal{S} \subseteq \mathcal{C}$, and suppose that there is an interior symmetry group $\Sigma_{\mathcal{S}}$. Let $f$ be a $G$-admissible vector field. In general, $f$ is not $\Sigma_{\mathcal{S}}$-equivariant, and $L$ does not commute with $\Sigma_{\mathcal{S}}$. However, the block matrix $A$ defined in (4.4) does commute with $\Sigma_{\mathcal{S}}$, so there is a natural $\Sigma_{\mathcal{S}} \times \mathbf{S}^{1}$-action on $E(A)$, where $\mathbf{S}^{1}$ acts by $\exp (s A)$. Let $\Delta \subset \Sigma_{\mathcal{S}} \times \mathbf{S}^{1}$ be a subgroup. The spatial subgroup of $\Delta$ is $K=\Delta \cap \Sigma_{\mathcal{S}}$.

Definition 6.1 The subgroup $\Delta \subset \Sigma_{\mathcal{S}} \times \mathbf{S}^{1}$ is spatially $\mathbf{C}$-axial if

$$
\operatorname{dim} \operatorname{Fix}_{E_{i}(A)}(\Delta)=\operatorname{dim} \operatorname{Fix}_{E_{i}(A)}(K)=2
$$

where $K$ is the spatial subgroup of $\Delta$.

We require the following concept:

Definition 6.2 The ODE (4.3) undergoes a synchrony-breaking Hopf bifurcation at $x_{0}$ when $\lambda=0$ if:

(a) After rescaling time, the linearization $L=(d f)_{x_{0}}$ at $\lambda=0$ has eigenvalues $\pm i$ coming from $A$, as defined in Lemma $4.2(\mathrm{c})$.

(b) The eigenvalue crossing condition (4.5) holds.

We use the term 'synchrony-breaking' because the equilibrium state is assumed to be in $\operatorname{Fix}\left(\Sigma_{\mathcal{S}}\right)$ (and hence synchronous on each $\Sigma_{\mathcal{S}}$-orbit in $\mathcal{S}$ ), whereas the Hopf bifurcation critical eigenvectors (corresponding to a critical eigenvalue of $A$ in (4.4)) are assumed to be transverse to $\operatorname{Fix}\left(\Sigma_{\mathcal{S}}\right)$ (which leads to periodic solutions not in $\operatorname{Fix}\left(\Sigma_{\mathcal{S}}\right)$ and hence with less synchrony).

The generalization of the Equivariant Hopf Theorem is:

Theorem 6.3 Consider the coupled cell system (4.3). Let $\mathcal{S}$ be a subset of cells with interior symmetry group $\Sigma_{\mathcal{S}}$, and let $x_{0} \in \operatorname{Fix}\left(\Sigma_{\mathcal{S}}\right)$ be an equilibrium of $f$. Assume that a synchronybreaking Hopf bifurcation occurs at $x_{0}$ when $\lambda=0$. Let $\Delta \subset \Sigma_{\mathcal{S}} \times \mathbf{S}^{1}$ be a spatial $\mathbf{C}$-axial subgroup. Then generically there exists a family of periodic solutions of (4.3), bifurcating from $\left(x_{0}, 0\right)$ and having period near $2 \pi$, that is synchronous on any two cells in $\mathcal{S}$ lying in 
the same K-orbit. Moreover, to lowest order in the bifurcation parameter $\lambda$, the solution $x(t)$ is of the form

$$
x(t) \approx u(t)+w(t)
$$

where $u(t)=e^{t L} u_{0}$ is synchronous on $\Sigma_{\mathcal{S}}$ group orbits of cells in $\mathcal{S}$ and $w(t)=e^{t L} v_{0}$ has exact $\Delta$ spatio-temporal symmetries on cells in $\mathcal{S}$.

Proof We adapt the proof of the Equivariant Hopf Theorem [27], which uses LiapunovSchmidt reduction, to the context of a synchrony-breaking Hopf bifurcation that breaks interior symmetry. Let $\mathcal{C}_{2 \pi}(P)$ be the loop space consisting of all continuous $2 \pi$-periodic functions from $\mathbf{S}^{1}$ into $P$, with the $C^{0}$ norm. The Hopf Theorem concerns periodic solutions to differential equations near a point where the Jacobian matrix has purely imaginary eigenvalues. We have rescaled time so that those purely imaginary eigenvalues are $\pm i$, so we look for periodic solutions with period near $2 \pi$. By introducing a perturbed period parameter $\tau$ we can rescale time again, from $t$ to $s=(1+\tau) t$, and consider $2 \pi$-periodic solutions to the equation

$$
\mathcal{F}(v(s), \lambda, \tau) \equiv(1+\tau) \frac{d v}{d s}-f(v(s), \lambda)=0
$$

Near the trivial equilibrium $(0,0,0)$ these solutions are zeros of the mapping

$$
\mathcal{F}: \mathcal{C}_{2 \pi}^{1}(P) \times \mathbf{R} \times \mathbf{R} \rightarrow \mathcal{C}_{2 \pi}(P)
$$

defined in $(6.3)$, where $\mathcal{C}_{2 \pi}^{1}(P)$ consists of the continuously differentiable functions in $\mathcal{C}_{2 \pi}(P)$ with the $C^{1}$ norm.

The linearization of $\mathcal{F}$ about the origin is

$$
\mathcal{L}(v(s))=\frac{d v}{d s}-L v(s)
$$

and $\operatorname{ker}(\mathcal{L})$ consists of all functions $v(s)=\operatorname{Re}\left(e^{i s} v\right)$ where $v$ is an eigenvector of $L$ associated to the eigenvalue $i$.

As is well known [27], the operator $\mathcal{F}$ is $\mathbf{S}^{1}$-equivariant with respect to the phase shift action of $\mathbf{S}^{1}$ on loop space. In the standard Hopf Theorem, the kernel $\operatorname{ker}(\mathcal{L})$ is two-dimensional, and Liapunov-Schmidt reduction in the presence of symmetry leads to a reduced equation that can be solved for a unique branch of $2 \pi$-periodic solutions as long as the eigenvalue crossing condition is valid.

In the equivariant context, however, $\operatorname{ker}(\mathcal{L})$ may be high-dimensional. The proof of the Equivariant Hopf Theorem proceeds by restricting the Liapunov-Schmidt reduced equation to $\operatorname{Fix}(\Delta)$. Since $\Delta$ is $\mathbf{C}$-axial, $\operatorname{Fix}(\Delta)$ is two-dimensional, so we can complete the proof as in the standard Hopf Theorem. That approach does not work in the context of interior symmetries (since the fixed-point subspace of $\Delta$ in loop space need not be $\mathcal{F}$-invariant). However, the fixed-point subspace of the spatial subgroup $K$ is $\mathcal{F}$-invariant (by Proposition 3.3). Since $\Delta$ is spatially $\mathbf{C}$-axial, $\operatorname{Fix}(K)$ is two-dimensional, and the proof now proceeds as in the equivariant case. 
The form of the eigenvectors in Lemma 4.2(c) implies that at linear level the solution of (4.3), with period near $2 \pi$, is of the form

$$
v(t)=w(t)+u(t)
$$

where $w(t) \in \operatorname{Fix}_{W}(\Delta)$ and $u(t) \in \operatorname{Fix}\left(\Sigma_{\mathcal{S}}\right)$. In particular, $w(t)$ has spatio-temporal symmetry $\Delta$ on cells in $\mathcal{S}$, and $u(t)$ is synchronous on $\Sigma_{\mathcal{S}}$ group orbits of cells in $\mathcal{S}$.

Remark 6.4 (1) The theorem asserts no restrictions on $v_{j}(t)$ when $j \notin \mathcal{S}$.

(2) The structure in (6.2) does impose genuine restrictions. Suppose, for the sake of illustration, that $\Delta$ defines a rotating wave. That is,

$$
\Delta=\langle(\zeta, \theta)\rangle \subseteq \Sigma_{\mathcal{S}} \times \mathbf{S}^{1}
$$

where $\zeta$ is a $k$-cycle on $\mathcal{S}$, with $|\mathcal{S}|=k$, and $\theta=2 \pi / k$ for the period $2 \pi$ case. Then on $\mathcal{S}=\{0, \ldots, k-1\}$ we have

$$
w_{0}(t)=w_{1}(t+2 \pi / k)=\cdots=w_{k-1}(t+2(k-1) \pi / k)
$$

and

$$
u_{0}(t)=u_{1}(t)=\cdots=u_{k-1}(t)
$$

for all $t$. To first order in $\lambda$, near bifurcation, the wave $v(t)$ satisfies

$$
v_{j}(t) \approx w_{j}(t)+u_{j}(t)
$$

If we define

$$
y_{j}(t)=v_{j+1}(t)-v_{j}(t)=w_{j+1}(t)-w_{j}(t)
$$

(subscripts modulo $k$ ) then

$$
y_{j}(t) \approx y_{0}(t+2 \pi j / k)
$$

is an approximate rotating wave. For a general periodic state, no such relation on differences holds.

Example 6.5 Consider the four-cell network whose diagram is Figure 2. Set $\mathcal{S}=\{1,2,3\}$; observe that on the right is a $\mathbf{Z}_{3}$-symmetric network and on the left is an network with one missing connection and $\mathbf{Z}_{3}$ interior symmetry. We compare Hopf bifurcations in the two networks.

Symmetry-breaking Hopf bifurcation that breaks $\mathbf{Z}_{3}$ symmetry leads to periodic solutions that are exact rotating waves in cells 1-3, and triple frequency motion in cell 4 . Interior symmetry-breaking Hopf bifurcation leads to periodic solutions that, to first order, are the sum of a periodic rotating wave and a periodic state that is synchronous on $\mathcal{S}$; in addition, cell 4 has the same frequency as cells $1-3$. Therefore, the time series $x_{1}-x_{2}, x_{2}-x_{3}, x_{3}-x_{1}$ form an approximate rotating wave. 



Figure 2: (Left) $\mathbf{Z}_{3}$ interior symmetry in a four-cell example. (Right) $\mathbf{Z}_{3}$-symmetric network.

In the interior symmetry case we test these conclusions using one-dimensionsal cells with equations

$$
\begin{aligned}
& \dot{x}_{1}=-x_{1}+0.5 x_{1}^{2}-x_{1}^{3}+\lambda x_{3}+2 x_{4} \\
& \dot{x}_{2}=-x_{2}+0.5 x_{2}^{2}-x_{2}^{3}+\lambda x_{1}+2 x_{4} \\
& \dot{x}_{3}=-x_{3}+0.5 x_{3}^{2}-x_{3}^{3}+\lambda x_{2}+2 x_{4} \\
& \dot{x}_{4}=-x_{4}+0.5 x_{4}^{2}-x_{4}^{3}-\left(x_{1}+x_{3}\right)
\end{aligned}
$$

where $\lambda=-2.05$. In the symmetry case we adapt (6.4) by changing the coupling term in the equation for $\dot{x}_{4}$ to $-\left(x_{1}+x_{2}+x_{3}\right)$.

A simulation is given in Figure 3. The upper panels show superimposed time series from all cells; cell 4 in black is the smallest in amplitude. The lower panels show the triple frequency exhibited by cell 4 in the symmetric case. In Figure 4 we see the rotating wave in the symmetric case on the right and the approximate rotating wave in coordinates $x_{1}-x_{2}$, $x_{2}-x_{3}, x_{3}-x_{1}$ on the left.

\section{Acknowledgments}

This work was supported in part by NSF Grants DMS-0071735 and DMS-0244529 and ARP Grant 003652-0032-2001.

\section{References}

[1] H. Brandt. Über eine Verallgemeinerung des Gruppenbegriffes, Math. Ann. 96 (1927) 360-366.

[2] P.C. Bressloff, J.D. Cowan, M. Golubitsky, and P.J. Thomas. Scalar and pseudoscalar bifurcations: pattern formation in the visual cortex, Nonlinearity 14 (2001) 739-775.

[3] P.C. Bressloff, J.D. Cowan, M. Golubitsky, P.J. Thomas, and M.C. Wiener. Geometric visual hallucinations, Euclidean symmetry, and the functional architecture of striate cortex, Phil. Trans. Royal Soc. B 356 (2001) 299-330. 

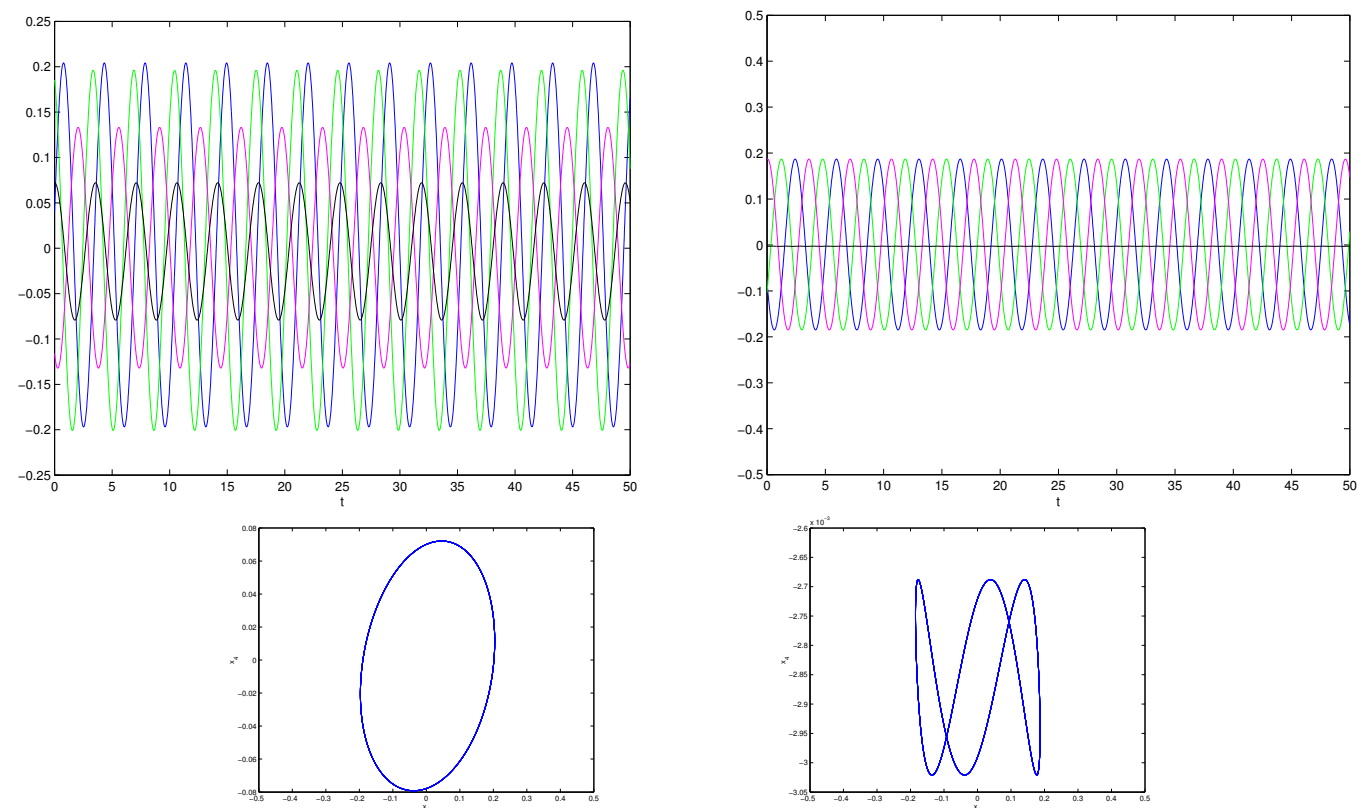

Figure 3: (Left) $\mathbf{Z}_{3}$ interior symmetry in a four-cell example. (Right) $\mathbf{Z}_{3}$-symmetric network. Upper panels show time series from all cells; lower panels show $x_{1}$ versus $x_{4}$.

[4] P.C. Bressloff, J.D. Cowan, M. Golubitsky, P.J. Thomas, and M.C. Wiener. What geometric visual hallucinations tell us about the visual cortex, Neural Computation 14 (2002) 473-491.

[5] S. Boccaletti, L.M. Pecora, and A. Pelaez. Unifying framework for synchronization of coupled dynamical systems, Phys. Rev E 63 (2001) 066219.

[6] R. Brown. From groups to groupoids: a brief survey, Bull. London Math. Soc. 19 (1987) $113-134$.

[7] Buono, P-L. A Model of Central Pattern Generators for Quadruped Locomotion, Ph.D Dissertation, U Houston 1998.

[8] Buono, P-L. Models of Central Pattern Generators for Quadruped Locomotion: II. Secondary Gaits, J. Math. Biol. 42 No. 4 (2001) 327-346.

[9] P.L. Buono and M. Golubitsky. Models of central pattern generators for quadruped locomotion: I. primary gaits, J. Math. Biol. 42 No. 4 (2001) 291-326.

[10] J.J. Collins and I. Stewart. Symmetry-breaking bifurcation: a possible mechanism for 2:1 frequency-locking in animal locomotion, J. Math. Biol. 30 (1992) 827-838.

[11] J.J. Collins and I. Stewart. Hexapodal gaits and coupled nonlinear oscillator models, Biol. Cybern. 68 (1993) 287-298. 

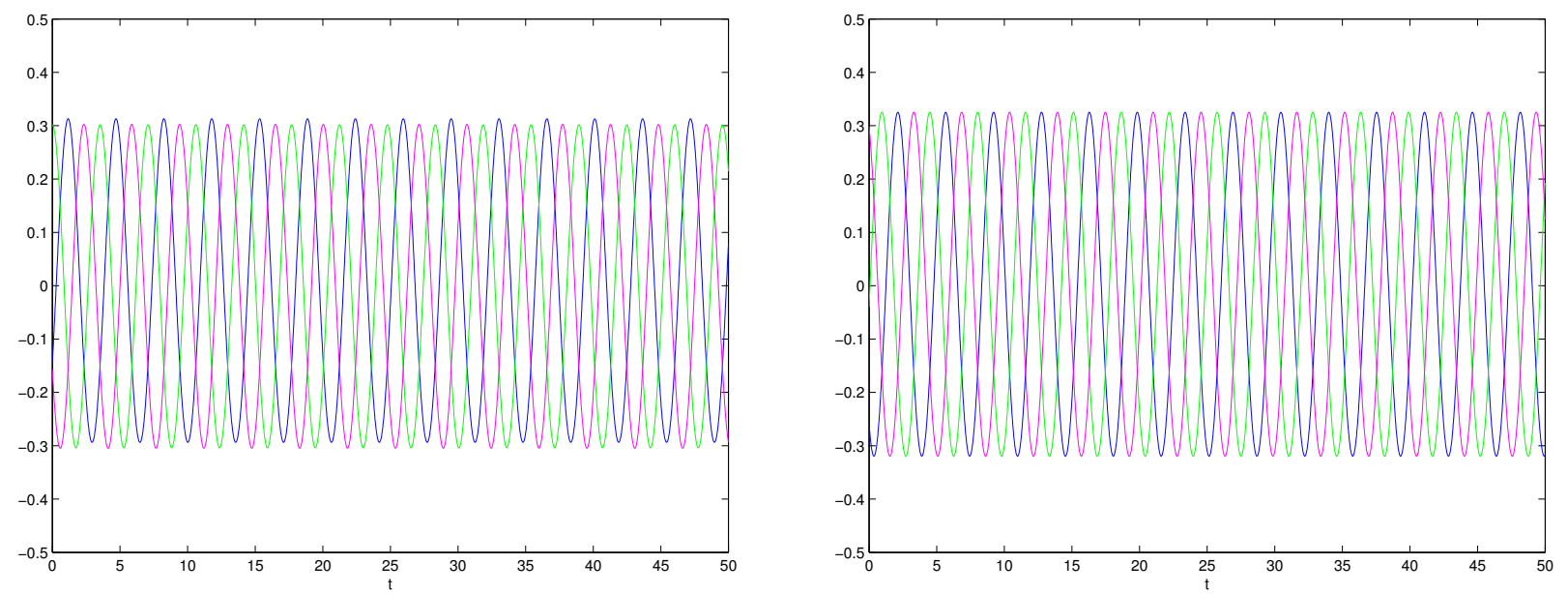

Figure 4: Simulations in cells 1-3 viewed in difference coordinates $x_{1}-x_{2}, x_{2}-x_{3}, x_{3}-x_{1}$. (Left) Approximate rotating wave in $\mathbf{Z}_{3}$ interior symmetry. (Right) Exact rotating wave in $\mathbf{Z}_{3}$-symmetric network.

[12] J.J. Collins and I. Stewart. Coupled nonlinear oscillators and the symmetries of animal gaits, J. Nonlin. Sci. 3 (1993) 349-392.

[13] J. Cohen and I. Stewart. Polymorphism viewed as phenotypic symmetry-breaking. In Nonlinear Phenomena in Physical and Biological Sciences (ed. S.K. Malik), Indian National Science Academy, New Delhi 2000, 1-67.

[14] A.P.S. Dias and I.Stewart. Secondary bifurcations in systems with all-to-all coupling, preprint, Math Dept., Porto 2002. Proc. Roy. Soc. London A. To appear.

[15] A.P.S. Dias and I.Stewart. Symmetry groupoids and admissible vector fields for coupled cell networks. To appear.

[16] B. Dionne, M. Golubitsky, and I. Stewart. Coupled cells with internal symmetry Part 1: wreath products, Nonlinearity 9 (1996) 559-574.

[17] B. Dionne, M. Golubitsky, and I. Stewart. Coupled cells with internal symmetry Part 2: direct products, Nonlinearity 9 (1996) 575-599.

[18] T.Elmhirst. Symmetry-breaking Bifurcations of $\mathbf{S}_{N^{-}}$-equivariant Vector Fields and Polymorphism, MSc Thesis, Mathematics Institute, University of Warwick 1998.

[19] T. Elmhirst. Symmetry and Emergence in Polymorphism and Sympatric Speciation, PhD Thesis, Mathematics Institute, University of Warwick 2001.

[20] T. Elmhirst. $\mathbf{S}_{N^{-}}$equivariant symmetry-breaking bifurcations, Internat. J. Bif. Chaos. To appear. 
[21] M. Golubitsky, M. Nicol, and I. Stewart. Some curious phenomena in coupled cell networks. Submitted.

[22] M. Golubitsky and D.G. Schaeffer. Singularities and Groups in Bifurcation Theory: Vol. 1. Applied Mathematical Sciences 69, Springer, New York 1985.

[23] M. Golubitsky and I. Stewart. The Symmetry Perspective: From Equilibrium to Chaos in Phase Space and Physical Space, Progress in Mathematics 200, Birkhäuser, Basel 2002.

[24] M. Golubitsky and I. Stewart. Patterns of oscillation in coupled cell systems. In: Geometry, Dynamics, and Mechanics: 60th Birthday Volume for J.E. Marsden (eds. P. Holmes, P. Newton, and A. Weinstein) Springer, New York 2002, 243-286.

[25] M. Golubitsky, I. Stewart, and A. Török. Patterns of synchrony in coupled cell networks with multiple arrows. Submitted.

[26] M. Golubitsky, I. Stewart, P.-L. Buono, and J.J. Collins. A modular network for legged locomotion, Physica D 115 (1998) 56-72.

[27] M. Golubitsky, I.N. Stewart and D.G. Schaeffer. Singularities and Groups in Bifurcation Theory: Vol. 2. Applied Mathematical Sciences 69, Springer, New York 1988.

[28] P.J. Higgins. Notes on Categories and Groupoids, Van Nostrand Reinhold Mathematical Studies 32, Van Nostrand Reinhold, London 1971.

[29] Y. Kuramoto. Chemical Oscillations, Waves, and Turbulence, Springer, Berlin 1984.

[30] L.M. Pecora and T.L. Carroll. Synchronization in chaotic systems, Phys. Rev. Lett. 64 (1990) 821-824.

[31] I. Stewart. Self-organization in evolution: a mathematical perspective. Proceedings of Nobel Symposium on Self-Organization, Stockholm 2002. To appear.

[32] I. Stewart. Speciation: a case study in symmetric bifurcation theory, Univ. Iagellonicae Acta Math. (2003). To appear.

[33] I. Stewart, T. Elmhirst, and J. Cohen. Symmetry-breaking as an origin of species. In Bifurcations, Symmetry, and Patterns, (eds. J.Buescu, S.B.S.D.Castro, A.P.S.Dias and I.S.Labouriau), Birkhäuser, Basel. To appear.

[34] I. Stewart, M. Golubitsky, and M. Pivato. Symmetry groupoids and patterns of synchrony in coupled cell networks, SIAM J. Appl. Dynam. Sys.. To appear.

[35] T.L. Vincent and T.L.S. Vincent. Evolution and control system design, IEEE Control Systems Magazine (October 2000) 20-35. 
[36] X.F. Wang. Complex networks: topology, dynamics and synchronization, Internat. J. Bif. Chaos 12 (2002) 885-916.

[37] D.J. Watts and S.H. Strogatz. Collective dynamics of 'small world' networks, Nature 393 440-442. 